\title{
A phase II study of S-1 in relapsed small cell lung cancer
}

\author{
KEITA KUDO, FUMIYOSI OHYANAGI, ATSUSHI HORIIKE, EISAKU MIYAUCHI, HISASHI TAHANAKA, \\ NORIKO YANAGITANI, RYOUTA SAITO, KYOUHEI KABURAKI, \\ TOSHIO SAKATANI, TAKESHI HORAI and MAKOTO NISHIO
}

\begin{abstract}
Thoracic Oncology Center, Cancer Institute Hospital, Japanese Foundation for Cancer Research, Koto-ku, Tokyo 135-8550, Japan
\end{abstract}

Received August 4, 2012; Accepted December 24, 2012

DOI: $10.3892 / \mathrm{mco} .2013 .67$

\begin{abstract}
S-1 is a new oral fluoropyrimidine derivative designed to enhance anticancer activity and reduce gastrointestinal toxicity. This phase II trial aimed to evaluate S-1 in patients with relapsed small cell lung cancer (SCLC). SCLC patients who had experienced treatment failure with $\geq 1$ prior chemotherapies were eligible. Patients were required to have an Eastern Cooperative Oncology Group (ECOG) performance status (PS) of 0-2 and adequate organ function. Treatment consisted of oral S-1 at $40 \mathrm{mg} / \mathrm{m}^{2}$ twice/day for 28 days every 6 weeks. Twenty-six patients were enrolled, $85 \%$ of whom were males. The median age was 68 years (range, 33-79) and $81 \%$ of the patients had a performance status of $0-1$, and $46 \%$ of the patients had relapse-sensitive SCLC. An objective response was obtained in only 1 patient (3.8\%), and the median progression-free survival (PFS) was 1.1 months. The median overall survival was 5.3 months, and the 1-year survival rate was $23 \%$. The most common grade $3 / 4$ toxicities included neutropenia (7.7\%), leukopenia (7.7\%), anemia (7.7\%), hyponatremia $(7.7 \%)$, rash $(7.7 \%)$, infection $(7.7 \%)$ and diarrhoea (3.8\%). None of the patients developed febrile neutropenia and no deaths were attributed to treatment. In conclusion, S-1 has minimal single-agent activity in relapsed SCLC.
\end{abstract}

\section{Introduction}

Lung cancer is the leading cause of mortality in Japan, and small cell lung cancer (SCLC) accounts for $15-20 \%$ of all the types of lung cancer (1). Although SCLC is an extremely chemosensitive disease, it is ultimately fatal in the majority of patients. Several anticancer agents tested over the last three decades have demonstrated some activity, but there have been only minimal improvements in the treatment of extensive SCLC (2).

Correspondence to: Dr Makoto Nishio, Thoracic Oncology Center, Cancer Institute Hospital, Japanese Foundation for Cancer Research, Ariake 3-8-31, Koto-ku, Tokyo 135-8550, Japan

E-mail:mnishio@jfcr.or.jp

Key words: lung cancer, small cell lung cancer, S-1, relapse
Based on the findings of a randomized trial comparing topotecan with cyclophosphamide, doxorubicin and vincristine in patients with relapse-sensitive SCLC, topotecan was considered to be a standard treatment in the second-line setting (3). However, the response rate ranged from 7 to $21 \%$, with a median survival time of only 6 months (3). Therefore, additional options are needed for patients with relapsed SCLC.

$\mathrm{S}-1$ is a novel oral fluoropyrimidine anticancer agent designed to enhance anticancer activity and reduce gastrointestinal toxicity. It is a combination of an oral fluoropyrimidine (tegafur), a dihydropyrimidine dehydrogenase (DPD) inhibitor (5-chloro-2,4-dihydroxypyridine), and an orotate phosphoribosyl transferase inhibitor (potassium oxonate) (4). Although 5-fluorouracil (5-FU) was thought to be inactive against non-small cell lung cancer (NSCLC) and SCLC $(5,6)$, single-agent $\mathrm{S}-1$ has been shown to provide one of the highest response rates against metastatic NSCLC and previously treated NSCLC (7). In addition, the combination of S-1 and cisplatin or carboplatin has been evaluated in Japanese phase III studies. The results of a phase III trial demonstrated the non-inferiority of carboplatin/S-1 compared to carboplatin/paclitaxel in terms of overall survival time (OS) (8). Most of the agents that are active against NSCLC have been tested and have also exhibited activity against SCLC. However, the activity of S-1 against SCLC has not been determined. Therefore, this study aimed to examine the activity of S-1 in patients with relapsed SCLC.

\section{Materials and methods}

Study subject criteria. Eligible patients had histologically or cytologically confirmed SCLC. The patients were $\geq 20$ years, had measurable disease, an Eastern Cooperative Oncology Group (ECOG) performance status (PS) of 0-2, and adequate bone marrow, kidney and liver functions. Patients were required to have received at least 1 prior chemotherapy regimen (including 1 regimen containing a platinum agent). Relapse-refractory and -sensitive patients were eligible. Patients who had undergone radiation therapy were required to have had their last treatment at least 14 days prior to registration in the protocol.

Patients were excluded due to symptomatic central nervous system metastasis, uncontrolled pleural effusion, pregnancy or lactation, the use of phenytoin, warfarin or flucytosine, 
or medical problems of marked severity. Patients previously treated with S-1 were not eligible. The treatment protocol was approved by the Investigational Review Board of the Cancer Institute Hospital (Tokyo, Japan). Patients provided written informed consent.

Study design and sample size. This study was an open-label, single-institution, phase II study of the single-agent S-1 for patients with previously treated SCLC. Simon's two-stage optimal design was chosen to determine the total number of patients required for this phase II study. A response rate of $25 \%$ was set for the target activity level, with $5 \%$ as the lowest response rate [objective response rate (ORR)] of interest. The study was designed to have $90 \%$ power to accept and $10 \%$ significance to reject the hypothesis. The planned sample size was fixed at 26 patients without test power consideration. If $>2$ responses were observed by the end of the study, further investigation of the drug was considered necessary.

Treatment plan. Treatment consisted of oral administration of $\mathrm{S}-1$ at $40 \mathrm{mg} / \mathrm{m}^{2}$ twice/day for 28 days, every 6 weeks. The actual dose of S-1 was selected as follows: for a patient with body surface area (BSA) $<1.25 \mathrm{~m}^{2}, 40 \mathrm{mg}$ twice/day; for BSA of $1.25 \mathrm{~m}^{2}$ but $<1.5 \mathrm{~m}^{2}, 50 \mathrm{mg}$ twice/day; and for BSA $1.5 \mathrm{~m}^{2}$, $60 \mathrm{mg}$ twice/day.

Statistical analysis. Intention-to-treat analysis considering the patients was performed. The safety analysis was based on the patients that had received any dose of study treatment. The primary endpoint was best ORR according to the Response Evaluation Criteria in Solid Tumors. Secondary efficacy endpoints were overall survival time (OS), progression-free survival time (PFS) and toxicity profile. OS and PFS were estimated using the Kaplan-Meier method. Toxicities were graded according to the Common Toxicity Criteria version 3.0.

\section{Results}

Patient characteristics. Between September, 2006 and May, 2008, 26 patients were enrolled in this study. Patient characteristics are summarized in Table I. The median age was 68 years (range, 33-79), and $81 \%$ of the patients had an ECOG PS of $0-1$. The median number of previous chemotherapy treatment regimens was 2 (range, 1-3) and 54\% of the patients received $\geq 2$ regimens. There were 12 relapse-sensitive patients (46\%) and 14 relapse-refractory patients (54\%).

Treatment administration. The median number of S-1 cycles administered was 2 (range, 1-5). Twenty patients received 1 cycle due to disease progression (16 patients) or treatment-related toxicities (dermatitis and infection in 2 patients, respectively). No dose delays or modifications were required. The patients were included in the efficacy analyses.

Response and survival. Response to treatment and survival of patients is shown in Table II. Among the relapse-sensitive patients, partial response was achieved in 1 (8.3\%) and stable disease in 4 patients (33.3\%). Among the relapse-refractory patients, no patient $(0 \%)$ had a partial response and 6 patients $(42.8 \%)$ achieved stable disease. Progressive disease as the
Table I. Patient characteristics.

\begin{tabular}{lr}
\hline Characteristics & Value \\
\hline No. of patients & 26 \\
Median age (years), n (range) & $68(33-79)$ \\
Gender, n (\%) & \\
Male & $22(85)$ \\
Female & $4(15)$ \\
Performance status, n $(\%)$ & \\
0 & $16(62)$ \\
1 & $5(19)$ \\
2 & $5(19)$ \\
Prior chemotherapy regimens, $\mathrm{n}(\%)$ & \\
1 & $12(46)$ \\
2 & $9(35)$ \\
3 & $5(19)$ \\
Relapse-sensitive cases, $\mathrm{n}(\%)$ & $12(46)$ \\
Relapse-refractory cases, $\mathrm{n}(\%)$ & $14(54)$ \\
\hline
\end{tabular}

best response was noted in $7(58.3 \%)$ of the relapse-sensitive patients and in $8(57.1 \%)$ of the relapse-refractory patients. The median time to disease progression was 1.1 months [95\% confidence interval (CI), 0.9-1.2 months]. The median overall survival was 5.3 months (95\% CI, 2.9-7.7 months), while the 1-year survival rate was $23 \%$.

Toxicity. Treatment-related toxicity is shown in Table III. In general, S-1 was well-tolerated. No patient developed febrile neutropenia or died due to the treatment.

\section{Discussion}

This phase II study was the first study to evaluate the activity of single-agent S-1 against relapsed SCLC. However, poor response rates were detected, and the majority of patients had early progressive disease. Single-agent S-1 has minimal activity in patients with previously treated SCLC, including those with a previous chemotherapy-sensitive disease.

Results similar to S-1 have been reported for another agent, pemetrexed. Since several clinical studies on NSCLC demonstrated positive findings, pemetrexed has also been thought to act against NSCLC (9). The efficacy of pemetrexed against SCLC has been examined in several studies (10-12). However, the results of those studies have been negative.

S-1 and pemetrexed have common characteristics. The primary cytotoxic mechanism of both S-1 and pemetrexed is the inhibition of thymidylate synthase (TS) $(13,14)$. Recent clinical trials have demonstrated that pemetrexed efficacy varied according to the histologic types of lung cancer $(9,11,12)$.

A possible explanation may involve TS expression levels in different histologic types of lung cancer, since preclinical data have shown that overexpression of TS correlates with reduced sensitivity to pemetrexed and 5-FU derivatives $(15,16)$. The baseline expression of TS is markedly higher in squamous cell carcinoma compared to adenocarcinoma $(15,16)$. In addition, 
Table II. Response to treatment, time to progression and overall survival of patients.

Patients, n (\%)

\begin{tabular}{lccc} 
Response & Relapse-sensitive $(\mathrm{n}=12)$ & Relapse-refractory $(\mathrm{n}=14)$ & Total $(\mathrm{n}=26)$ \\
\cline { 2 - 4 } $\begin{array}{l}\text { Best response to treatment } \\
\text { Complete }\end{array}$ & $0(0)$ & $0(0)$ & $0(0)$ \\
Partial & $1(8.3)$ & $0(0)$ & $1(3.8)$ \\
Stable disease & $4(33.3)$ & $6(42.8)$ & $10(38)$ \\
Progressive disease & $7(58.3)$ & $8(57.1)$ & $15(58)$ \\
Objective response rate & $1(8.3)$ & $0(0)$ & $1(3.8)$ \\
Disease control rate & $5(41.6)$ & $6(42.8)$ & $11(42.3)$ \\
Median time to progression (days) & 34 & 32 & 33 \\
Median overall survival (months) & 8.4 & 4.0 & 5.3 \\
\hline
\end{tabular}

Table III. Haematological and non-haematological toxicities.

\begin{tabular}{lrrrrr}
\hline & \multicolumn{5}{c}{ Grade } \\
\cline { 2 - 4 } Toxicity & 1 & 2 & 3 & 4 & $3 / 4(\%)$ \\
\hline Haematological & & & & & \\
Leukopenia & 9 & 6 & 2 & 0 & 7.7 \\
Neutropenia & 9 & 2 & 1 & 1 & 7.7 \\
Febrile neutropenia & 0 & 0 & 0 & 0 & 0 \\
Anaemia & 13 & 5 & 1 & 1 & 7.7 \\
Thrombopenia & 2 & 0 & 2 & 0 & 7.7 \\
Non-haematological & & & & & \\
Aspartate aminotransferase & 8 & 0 & 1 & 0 & 3.8 \\
Alanine aminotransferase & 3 & 2 & 1 & 0 & 3.8 \\
Hyponatremia & 16 & - & 0 & 2 & 7.7 \\
Hypokalemia & 0 & 0 & 2 & 0 & 7.7 \\
Anorexia & 20 & 6 & 0 & 0 & 0 \\
Nausea & 6 & 4 & 0 & 0 & 0 \\
Diarrhoea & 5 & 3 & 1 & 0 & 3.8 \\
Rash & 4 & 3 & 2 & 0 & 7.7 \\
Malaise & 15 & 2 & 0 & 0 & 0 \\
Infection without neutropenia & 0 & 2 & 2 & 0 & 7.7 \\
\hline
\end{tabular}

TS expression in neuroendocrine tumors has been examined, and higher TS expression was observed in SCLC and large cell neuroendocrine carcinoma compared to other types of lung cancer $(17,18)$.

However, in contrast with pemetrexed, findings of phase II and III trials of S-1 against NSCLC did not demonstrate any obvious differences in the efficacy of $\mathrm{S}-1$ against squamous and non-squamous NSCLC (7).

The reason for this discrepancy between pemetrexed and S-1 is unclear. S-1 may be able to inhibit higher levels of TS compared to pemetrexed. However, TS activity in SCLC may be considerably higher than S-1 can inhibit, since expression of TS in SCLC was shown to be markedly higher compared to TS expression in squamous cell carcinoma (17).

In addition, DPD inhibition may play an important role in NSCLC compared to SCLC. Several studies have demonstrated that 5-FU sensitivity is affected by DPD expression, which is an enzyme in NSCLC affecting 5-FU catabolism (19-22).

In conclusion, $\mathrm{S}-1$ monotherapy is well-tolerated but has low activity in patients with relapsed previously treated SCLC patients, including those with a previous chemotherapysensitive disease. Findings of this study have shown that S-1 has minimal single-agent activity in relapsed SCLC.

\section{References}

1. Stupp R, Monnerat C, Turrisi AT II, Perry MC and Leyvraz S: Small cell lung cancer: state of the art and future perspectives. Lung Cancer 45: 105-117, 2004.

2. Chute JP, Chen T, Feigal E, Simon R and Johnson BE: Twenty years of phase III trials for patients with extensive-stage small-cell lung cancer: perceptible progress. J Clin Oncol 17: 1794-1801, 1999.

3. Hurwitz JL, McCoy F, Scullin P and Fennell DA: New advances in the second-line treatment of small cell lung cancer. Oncologist 14: 986-994, 2009.

4. Shirasaka T, Yamamitsu S, Tsuji A and Taguchi T: Conceptual changes in cancer chemotherapy: from an oral fluoropyrimidine prodrug, UFT, to a novel oral fluoropyrimidine prodrug, S-1, and low-dose FP therapy in Japan. Invest New Drugs 18: 315-329, 2000.

5. Citron ML, Modeas C, Propert K, Goutsou M and Green MR: Phase II trial of high-dose 24-hour continuous intravenous 5-fluorouracil for advanced non-small cell lung cancer: a Cancer and Leukemia Group B study. Cancer Invest 10: 215-219, 1992.

6. Stewart DJ, Dahrouge S, Soltys KM and Evans WK: A phase II study of 5-fluorouracil plus high-dose folinic acid in the treatment of recurrent small cell lung cancer. Am J Clin Oncol 18: 130-132, 1995.

7. Yamamoto N, Yamanaka T, Ichinose Y, et al: Pooled analysis of $\mathrm{S}-1$ trials in non-small cell lung cancer according to histological type. Anticancer Res 30: 2985-2990, 2010.

8. Okamoto I, Yoshioka H, Morita S, et al: Phase III trial comparing oral S-1 plus carboplatin with paclitaxel plus carboplatin in chemotherapy-naive patients with advanced non-small-cell lung cancer: results of a west Japan oncology group study. J Clin Oncol 28: 5240-5246, 2010.

9. Scagliotti G, Hanna N, Fossella F, et al: The differential efficacy of pemetrexed according to NSCLC histology: a review of two Phase III studies. Oncologist 14: 253-263, 2009. 
10. Socinski MA, Raju RN, Neubauer M, et al: Pemetrexed in relapsed small-cell lung cancer and the impact of shortened vitamin supplementation lead-in time: results of a phase II trial. J Thorac Oncol 3: 1308-1316, 2008.

11. Jalal S, Ansari R, Govindan R, et al: Pemetrexed in second line and beyond small cell lung cancer: a Hoosier Oncology Group phase II study. J Thorac Oncol 4: 93-96, 2009.

12. Grønberg BH, Bremnes RM, Aaseb $\varnothing \mathrm{U}$, et al: A prospective phase II study: high-dose pemetrexed as second-line chemotherapy in small-cell lung cancer. Lung Cancer 63: 88-93, 2009.

13. Shirasaka T, Shimamato Y, Ohshimo H, et al: Development of a novel form of an oral 5-fluorouracil derivative (S-1) directed to the potentiation of the tumor selective cytotoxicity of 5-fluorouracil by two biochemical modulators. Anticancer Drugs 7: 548-557, 1996.

14. Adjei AA: Pemetrexed (Alimta): a novel multitargeted antifolate agent. Expert Rev Anticancer Ther 3: 145-156, 2003.

15. Hanauske AR, Eismann U, Oberschmidt O, et al: In vitro chemosensitivity of freshly explanted tumor cells to pemetrexed is correlated with target gene expression. Invest New Drugs 25: 417-423, 2007.

16. Nakano J, Huang C, Liu D, et al: Evaluations of biomarkers associated with 5-FU sensitivity for non-small-cell lung cancer patients postoperatively treated with UFT. Br J Cancer 95: 607-615, 2006.
17. Ibe T, Shimizu K, Nakano T, et al: High-grade neuroendocrine carcinoma of the lung shows increased thymidylate synthase expression compared to other histotypes. J Surg Oncol 102: 11-17, 2010.

18. Ceppi P, Volante M, Saviozzi S, et al: Squamous cell carcinoma of the lung compared with other histotypes shows higher messenger RNA and protein levels for thymidylate synthase. Cancer 107: 1589-1596, 2006

19. Inoue K, Takao M, Watanabe F, et al: Role of dihydropyrimidine dehydrogenase inhibitory fluoropyrimidine against non-small cell lung cancer - in correlation with the tumoral expression of thymidylate synthase and dihydropyrimidine dehydrogenase. Lung Cancer 49: 47-54, 2005.

20. Beck A, Etienne MC, Cheradame S, et al: A role for dihydropyrimidine dehydrogenase and thymidylate synthase in tumour sensitivity to fluorouracil. Eur J Cancer 30A: 1517-1522, 1994.

21. Shintani Y, Ohta M, Hirabayashi H, et al: Thymidylate synthase and dihydropyrimidine dehydrogenase mRNA levels in tumor tissues and the efficacy of 5-fluorouracil in patients with non-small-cell lung cancer. Lung Cancer 45: 189-196, 2004.

22. Oguri T, Achiwa H, Bessho Y, et al: The role of thymidylate synthase and dihydropyrimidine dehydrogenase in resistance to 5-fluorouracil in human lung cancer cells. Lung Cancer 49: $345-351,2005$ 